\title{
Recruitment of an ancient branching program to suppress carpel development in maize flowers
}

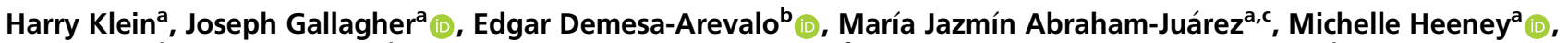

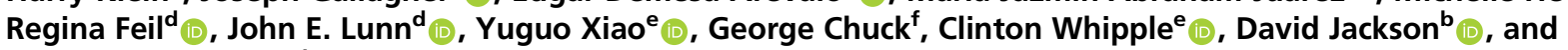 \\ Madelaine Bartlett ${ }^{a, 1}$ \\ aDepartment of Biology, University of Massachusetts Amherst, Amherst, MA 01003; bPlant Biology, Cold Spring Harbor Laboratory, Cold Spring Harbor, NY \\ 11724; ' Laboratorio Nacional de Genómica para la Biodiversidad, Unidad de Genómica Avanzada, Centro de Investigación y de Estudios Avanzados del \\ Instituto Politécnico Nacional, Guanajuato 36821, Mexico; ${ }^{\mathrm{d}}$ Max Planck Institute of Molecular Plant Physiology, 14476 Potsdam-Golm, Germany; ${ }^{e}$ Department \\ of Biology, Brigham Young University, Provo, UT 84692; and ${ }^{\mathrm{f} P l a n t}$ Gene Expression Center, University of California, Berkeley, CA 94710
}

Edited by Elizabeth Kellogg, Donald Danforth Plant Science Center, St. Louis, MO; received September 6, 2021; accepted November 16, 2021

Carpels in maize undergo programmed cell death in half of the flowers initiated in ears and in all flowers in tassels. The HD-ZIP I transcription factor gene GRASSY TILLERS1 (GT1) is one of only a few genes known to regulate this process. To identify additional regulators of carpel suppression, we performed a gt1 enhancer screen and found a genetic interaction between gt1 and ramosa3 (ra3). RA3 is a classic inflorescence meristem determinacy gene that encodes a trehalose-6-phosphate (T6P) phosphatase (TPP). Dissection of floral development revealed that ra3 single mutants have partially derepressed carpels, whereas gt1;ra3 double mutants have completely derepressed carpels. Surprisingly, gt1 suppresses ra3 inflorescence branching, revealing a role for gt 1 in meristem determinacy. Supporting these genetic interactions, GT1 and RA3 proteins colocalize to carpel nuclei in developing flowers. Global expression profiling revealed common genes misregulated in single and double mutant flowers, as well as in derepressed gt1 axillary meristems. Indeed, we found that ra3 enhances gt1 vegetative branching, similar to the roles for the trehalose pathway and GT1 homologs in the eudicots. This functional conservation over $\sim 160$ million years of evolution reveals ancient roles for GT1like genes and the trehalose pathway in regulating axillary meristem suppression, later recruited to mediate carpel suppression. Our findings expose hidden pleiotropy of classic maize genes and show how an ancient developmental program was redeployed to sculpt floral form.

programmed cell death | RAMOSA3 (RA3) | trehalose 6-phosphate GRASSY TILLERS1 (GT1) | evolution of flower development

V ariation in development drives variation in organismal form. One important process in floral development and evolution is growth suppression in floral organs $(1,2)$. A prominent form of this suppression exists in the grass family (Poaceae). Most grass flowers initiate both carpel and stamen primordia, but selective suppression of these primordia has led to immense diversity in floral sexuality (3). This diversity is critical for patterns of gene flow in natural populations and fertile flower production in nature and agriculture, and it facilitates controlled crosses in breeding programs (4-6). Despite these important consequences to both evolution and agriculture, only a handful of genes are known to regulate floral sexuality in the grasses.

Floral sexuality has long been studied in Zea mays (maize), in which programmed cell death suppresses carpel development in all tassel flowers and in one of the two flowers in each ear spikelet $(7,8)$. Among the few characterized carpel suppression genes, most encode enzymes with roles in hormone metabolism and have pleiotropic effects on development and defense (9-15). This list includes several genes underlying the classic tasselseed $(t s)$ mutants, which exhibit extensive tassel feminization beyond carpel suppression $(9,10,12-14,16)$. Most of the cloned $t s$ mutants encode genes involved in jasmonic acid metabolism, whereas $t s 4$ encodes a microRNA that targets $T s 6$, a developmental regulator with multiple roles in flower and inflorescence development $(12,14)$.

Most genes that affect carpel suppression simultaneously affect other traits that differentiate tassels from ears, such as stamen development, bract (glume) morphology, and inflorescence morphology $(9-11,16)$. GRASSY TILLERS1 (GT1) is an exception. While $G T 1$ was defined by its role in regulating axillary branching, gt1 mutants have a weak carpel suppression phenotype in otherwise normal flowers and inflorescences (17). To find additional regulators of carpel suppression, we conducted an enhancer screen of gt 1 mutants and found a genetic interaction with the classic inflorescence determinacy gene, RAMOSA3 (RA3) (18). Our results reveal surprising pleiotropy and interactions between $g t 1$ and $r a 3$, which together regulate carpel suppression, meristem determinacy, and axillary meristem suppression.

\section{Results}

The rapunzel (rzl) Genes Suppress Carpels in Tassel and Ear Flowers. To identify genes that regulate carpel suppression with gt1, we conducted an ethyl methanesulfonate (EMS) enhancer screen, looking for mutants where carpel growth was derepressed in

\section{Significance}

Floral morphology is immensely diverse. One developmental process acting to shape this diversity is growth suppression. For example, grass flowers exhibit extreme diversity in floral sexuality, arising through differential suppression of stamens or carpels. The genes regulating this growth suppression and how they have evolved remain largely unknown. We discovered that two classic developmental genes with ancient roles in controlling vegetative branching were recruited to suppress carpel development in maize. Our results highlight the power of forward genetics to reveal unpredictable genetic interactions and hidden pleiotropy of developmental genes. More broadly, our findings illustrate how ancient gene functions are recruited to new developmental contexts in the evolution of plant form.

Author contributions: H.K., J.G., C.W., D.J., and M.B. designed research; H.K., J.G., E.D.-A., M.J.A.-J., M.H., R.F., J.E.L., Y.X., G.C., and M.B. performed research; H.K., J.G., R.F., J.E.L., and M.B. analyzed data; and H.K., J.G., and M.B. wrote the paper with assistance from coauthors.

The authors declare no competing interest.

This article is a PNAS Direct Submission.

This open access article is distributed under Creative Commons AttributionNonCommercial-NoDerivatives License 4.0 (CC BY-NC-ND)

${ }^{1}$ To whom correspondence may be addressed. Email: mbartlett@umass.edu.

This article contains supporting information online at http://www.pnas.org/lookup/ suppl/doi:10.1073/pnas.2115871119/-/DCSupplemental.

Published January 7, 2022. 
tassels. Maize flowers typically initiate three stamens and three carpel primordia, with stamen development suppressed in ear flowers and carpel development suppressed in tassel flowers (Fig. $1 A$ and $D$ ). When carpel growth is not suppressed, gynoecia, each consisting of three carpels, develop in tassel flowers, resulting in silks emerging from tassel spikelets (Fig. 1C). In gt1 single mutants, only $7 \%$ of scored tassel flowers developed long silks that emerged from spikelets $(>0.2 \mathrm{~cm}$, Fig. $1 B$ and $G)$. However, in the double mutants we identified in our screen, $100 \%$ of scored tassel flowers developed long silks (Fig. $1 C$ and $G$ ). We called this enhanced mutant phenotype rapunzel $(r z l)$, after the Grimm brothers' fairy tale character with long hair. Two of these $r z l$ mutants, $g t 1 ; r z l-3$ and $g t 1 ; r z l-4$, phenocopied one another and did not complement each other, indicating that $r z l-3$ and $r z l-4$ are allelic (Fig. $1 C$ and I; SI Appendix, Fig. $\mathrm{S} 1)$. Therefore, we focused many of our downstream analyses on either $r z l-3$ or $r z l-4$.

The $g t 1 ; r z l-3 / 4$ floral phenotype was specific to carpels and manifested in both tassels and ears. In contrast to most $t s$ mutants $(9,10,12,13)$, stamen development in gt1;rzl-3 and gt1;rzl-4 tassel flowers was not suppressed (Fig. 1; SI Appendix, Fig. S1 and Table S1). In normal ear spikelets, two flowers initiate, but only the upper flower completes development; the lower flower is suppressed (7). In gt 1;rzl-3 ears, carpel growth was derepressed in lower flowers, resulting in two fertile flowers per spikelet (Fig. $1 \mathrm{~F}$ and $H$; SI Appendix, Fig. S2). Thus, $r z l-3 / 4$ interacts with gt1 to disrupt carpel suppression while leaving other floral and inflorescence traits unaffected.

rzl-3 and rzl-4 Are Alleles of the Trehalose-6-Phosphate Phosphatase Gene, RAMOSA3. To identify the $r z l-3 / 4$ gene, we used both bulked segregant analysis coupled with whole genome shotgun sequencing (BSA-seq) and fine mapping (19). Our BSA-seq results revealed a broad peak between 160 and $180 \mathrm{Mbp}$ on chromosome seven that represented the $r z l-4$ mapping interval (Fig. 2A). The narrow peaks on chromosomes three and nine are likely because of differences between B73 laboratory stocks and the reference genome $(19,20)$. The broad region of homozygosity on chromosome one results from the introgression of gt1, which arose in A619 (17), into B73. Using fine mapping
(19), we reduced the chromosome seven mapping interval to a $\sim 230$-kbp region containing nine genes (Fig. $2 B$ ), only one of which contained a canonical EMS single nucleotide polymorphism (SNP) predicted to negatively affect gene function (21).

This EMS SNP was predicted to change a single amino acid (Ala337Thr) in the trehalose-6-phosphate phosphatase (TPP) encoded by $R A M O S A 3$ (RA3) (18). Ala337 is deeply conserved in TPP paralogs and contacts the active site in homology models (SI Appendix, Fig. S3). In addition, the substitution of a homologous amino acid in an RA3 paralog negatively affects protein function (22), suggesting that the rzl-4 SNP would impact RA3 function similarly. Because $r z l-4$ and $r z l-3$ were allelic, we sequenced $R A 3$ in $r z l-3$ mutants and found an EMS mutation at a likely splice acceptor site $5^{\prime}$ of exon 4 . Transcripts from this ra3 allele had an in-frame deletion of two active-site amino acid codons (SI Appendix, Fig. S2). Thus, both gt1;rzl-3 and $g t 1, r z l-4$ double mutants harbor alleles of $r a 3$ predicted to negatively affect gene function, indicating that $r z l-3$ and $r z l-4$ encode alleles of ra3. From now on, we will refer to these alleles as ra3-rzl3 and ra3-rzl4.

gt1 Suppresses ra3 Tassel and Ear Branching. Usually, ra3 mutants have branched ears and increased tassel branching due to indeterminate meristems that produce many spikelets on long branches $(18,22)$. Therefore, we were surprised to find unbranched ears in gt1;ra3-rzl3/4 double mutants and in ra3$r z l 3 / 4$ single mutants heterozygous at $g t 1$ (Fig. $1 F$ ). Given that gt1 is semidominant, the lack of ear branching in these mutants could have been caused by a second genetic interaction between gt1 and ra3, regulating meristem determinacy. The lack of ear branching may also have been the result of the genetic background (A619) used for EMS mutagenesis. Most characterization of ra3 has been in the B73 genetic background $(18,22)$, and background modifiers affect the ra3 ear determinacy phenotype (18)

To test for a genetic interaction between $g t 1$ and $r a 3$ independent of any A619 modifiers, we made a gt1;ra3 double mutant with a third, well-characterized allele of $\mathrm{ra3}$ ( $\mathrm{ra3}-\mathrm{fea} 1)$ in the B73 genetic background (18, 22) (Fig. 2). gt1;ra3-feal double mutants in B73 recapitulated the $r z l$ phenotype, with silks in
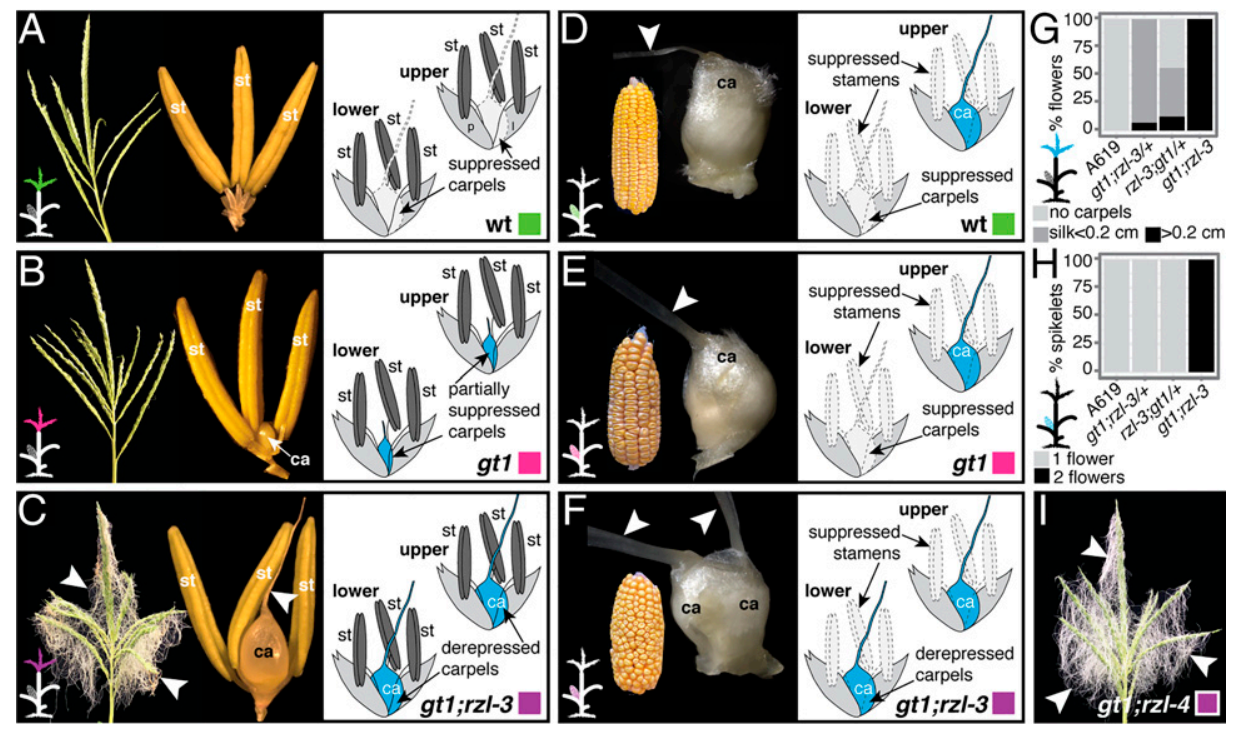

Fig. 1. gt1;rzl-3/4 double mutants have derepressed carpels in tassels and ears. $(A-C)$ Tassels and tassel flowers and $(D-F)$ ears and ear spikelets in $(A, D)$ wild-type (wt, A619), ( $B, E)$ gt1;rzl-3/+, and $(C, F)$ gt1;rzl-3. Upper and lower flowers are identical in tassel spikelets, and a single flower is shown in $A-C$. Arrowheads indicate silks. Palea and lemma removed from photographed flowers are shown in light gray in diagrams. (G, $H$ ) Carpels are derepressed in gt1;rzl-3 tassel and ear flowers. Categorization of $(G)$ tassel flowers (20 flowers from each of 5 individuals per genotype) and $(H)$ ear spikelets ( 50 spikelets from each of 5 individuals per genotype). (I) gt1;rzl-4 mutants phenocopy gt1;rzl-3 mutants. ca, carpels; I, lemma; p, palea; st, stamen. 

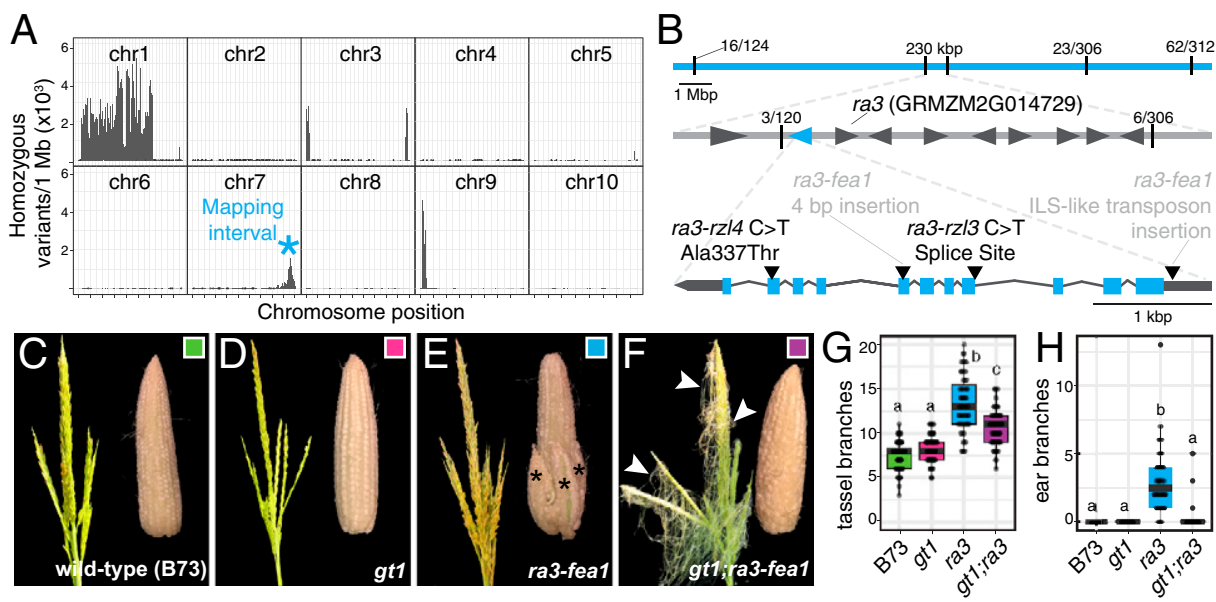

Fig. 2. $\quad r z l-3$ and $r z l-4$ are alleles of ramosa3 (ra3), which interacts genetically with gt1 in flowers and inflorescences. (A) BSA-seq mapped $r z l-4$ to chromosome 7. chr, chromosome. $(B)$ Mapping interval shown in detail. Black tick marks indicate genetic markers, and numbers above ticks indicate recombinants identified in fine mapping. ra3 alleles are identified here in black, ra3-fea1 from (18) in gray. (C-F) Tassels and ears from $(C)$ B73 (wt), $(D)$ gt1, (E) ra3-fea1 mutants, and $(F)$ gt1;ra3-fea 1 mutants, all in B73. Asterisks in $E$ mark ear branches, and arrowheads in $F$ indicate long, ectopic silks emerging from tassel flowers. $(G, H)$ gt1 suppresses ra3 inflorescence branching in both $(G)$ tassels and $(H)$ ears in the B73 genetic background. Tassel branches from 68 B73, 67 gt1, 59 ra3, and 66 gt1;ra3 individuals and ear branches from 26 B73, 30 gt1, 54 ra3, and 65 gt1;ra3 individuals were counted over two field seasons. Different letters indicate statistically significant differences $(P<0.001$, ANOVA, Tukey's post hoc).

tassel flowers, as in our gt1;ra3-rzl3 and gt1;ra3-rzl4 mutants (Fig. 2F). ra3-feal single mutants had branched ears and increased tassel branching, as expected (18). However, most gt1;ra3-feal double mutants lacked ear branches and had fewer tassel branches than $\mathrm{ra} 3$ single mutants (Fig. $2 \mathrm{C}-\mathrm{H}$ ), indicating a second genetic interaction between gt 1 and $\mathrm{ra3}$, regulating meristem determinacy. Thus, $R A 3$ and $G T 1$ act to regulate both meristem determinacy in inflorescences and carpel suppression in flowers.

ra3 Mutants Have a Carpel Suppression Phenotype and RA3 Colocalizes with GT1 in Carpel Nuclei. ra3-rzl3 mutants (ra3$r z l 3 ; g t 1 /+)$ had significantly more derepressed carpels in tassel flowers than A619 individuals (Fig. $1 G, P$ value $\ll 0.001, \chi^{2}$ test, two degrees of freedom). This could be because of gt1 semidominance (17) or because ra3 single mutants have a weak floral phenotype. Indeed, there have been hints of likely background-dependent ra3 floral phenotypes (23). To investigate the ra3 floral phenotype further, we followed tassel flower development in mutants using scanning electron microscopy.

In wild-type flowers, the two silk carpels are first visible as a raised line of tissue called the gynoecial ridge. In ear flowers, these silk carpels grow to enclose the third, ovulate carpel and fuse to form the stigma, called the silk in maize (7). In tassel flowers, carpels initiate but undergo programmed cell death shortly after the initiation of the gynoecial ridge $(8,24)$ (Fig. 3 $A-D)$. In contrast, in gt 1 single mutants, silk carpels continued to grow past the gynoecial ridge stage and formed a peak of tissue over the developing ovulate carpel (Fig. $3 E-H$ ). The tassel flowers of $\mathrm{ra} 3$ single mutants had carpels that resembled those of gt1 mutants: The two silk carpels formed a peak of tissue over the ovulate carpel but did not fuse laterally to enclose the ovulate carpel (Fig. $3 I-L$ ). These data indicate that carpel suppression is partially disrupted in both $\mathrm{ra} 3$ and gt 1 single mutants.

The carpels in gt1;ra3 double mutant flowers continued to grow well past the gynoecial ridge stage, eventually fusing to form silks (Fig. $3 M-P$; SI Appendix, Fig. S2). In addition, the gynoecia of gt1;ra3 double mutants closely resembled the gynoecia of wild-type ear florets, with two silk carpels and an ovulate carpel (Fig. $3 O$ and P; SI Appendix, Fig. S2). In contrast, other floral meristem determinacy mutants, such as
Z. mays agamous1 (zag1), bearded ear (bde), Tasselseed6/indeterminate spikelet1 (Ts6/ids1), drooping leaf1 (drl1), and drooping leaf2 (drl2), have indeterminate floral meristems, leading to disorganized gynoecia with extra primordia, many of which do not fuse to form silks $(12,25-29)$. Thus, our results indicate that the gt1;ra3 phenotype arises not because the floral meristems had become indeterminate but because floral organ suppression was disrupted.

The floral phenotypes of both single and double mutants led us to assess GT1 and RA3 localization in developing flowers, using immunofluorescence with custom antibodies. GT1 and RA3 both localized to the nuclei of carpel primordia in $1.5 \mathrm{~cm}$ tassels, prior to carpel growth suppression (Fig. 4A). We did

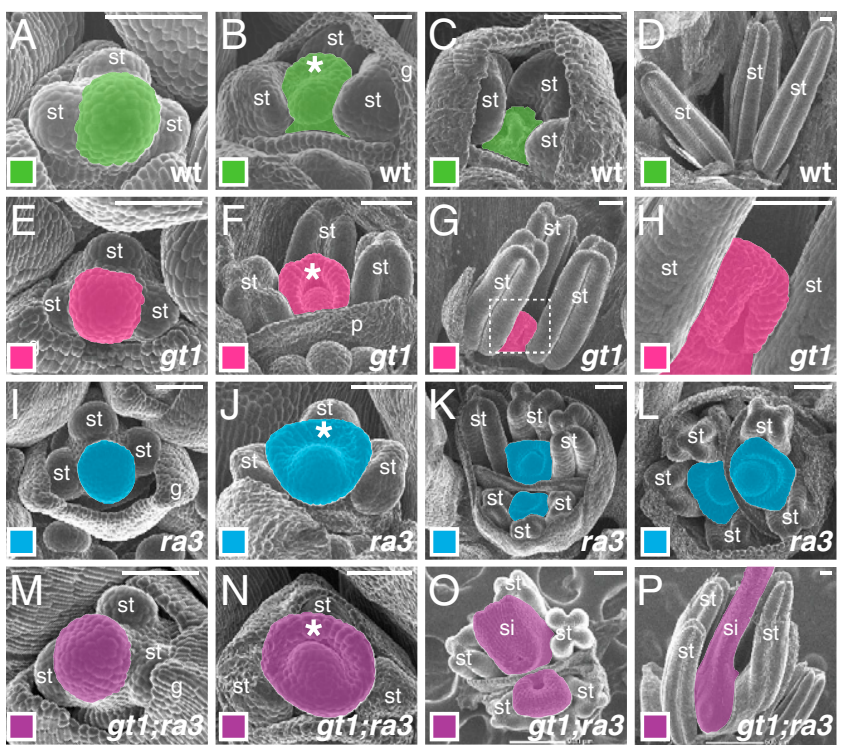

Fig. 3. ra3 single mutants exhibit a weak carpel derepression phenotype. Tassel flower development in $(A-D)$ wt (A619), (E-H) gt1, (I-L) ra3, and $(M-P)$ gt1;ra3 mutants is shown. Outlined region in $G$ is shown at higher magnification in $H$. Carpel primordia are false-colored, with no remains of carpels evident in $D$. g, glume; p, palea; si, silk; st, stamen; *gynoecial ridge. (Scale bar, $100 \mu \mathrm{m}$.) 
not detect GT1 protein in gt1 (B73) carpels, nor did we detect RA3 in ra3-feal carpels, which we used because ra3-feal is a known protein null allele (18). Importantly, both GT1 and RA3 were predominantly localized to the nuclei of subepidermal cells, where programmed cell death initiates in maize and its relatives in the Andropogoneae $(8,30)$. While $R A 3$ likely acts non-cell autonomously in regulating meristem determinacy $(18,31)$, the GT1 and RA3 localization patterns in tassel flowers suggest that both act cell autonomously in regulating carpel suppression.

GT1 and RA3 Regulate Genes Predicted to Mediate Programmed Cell Death. To identify the effectors of carpel suppression downstream of $G T 1$ and $R A 3$, we examined the transcriptional profiles of wild-type (A619), gt1, ra3, and gt1;ra3 tassels at two developmental timepoints: 1 ) prior to carpel suppression (presuppression, $0.8-1 \mathrm{~cm}$ tassels) and 2) during active carpel suppression (midsuppression, 1-2 cm tassels). We reasoned that genes that were 1) misexpressed in gt1;ra3 mutant flowers and 2) changed over wild-type carpel development represented the best carpel suppression gene candidates. We performed differential expression analyses on our sequencing data (32) and focused on the 73 genes that satisfied both of these conditions and were highly differentially expressed at the midsuppression stage ( $\mid$ fold change $\mid>2$, Fig. $4 B$ and $C$; Dataset S1). Most genes in this carpel suppression gene set that were downregulated in mutants were up-regulated over the course of development in A619 or vice versa (69/73 genes, or 95\%; Fig. $4 C)$. This pattern of regulation is consistent with a role for these genes in mediating the development of the $r z l$ phenotype. Notably, $62 / 73$ genes $(85 \%)$ were misexpressed in $g t 1$ or $r a 3$ single mutants, and 44/73 (60\%) were misexpressed in gt 1 and $\mathrm{ra3}$ single mutants. This suggests that the carpel suppression phenotype in gt1;ra3 double mutants is a matter of degree, not the result of an entirely different set of genes being misregulated in double versus single mutants.

Although the carpel suppression gene set was not enriched for specific gene ontology (GO) terms, it did contain several genes whose homologs in Arabidopsis thaliana (Arabidopsis) have roles in reactive oxygen species signaling, callose deposition, cell wall remodeling, and programmed cell death (Fig. $4 C$ and Table 1). For example, homologs of the Arabidopsis NAM, ATAF and CUC (NAC)-family transcription factor genes KIRA1 (GRMZM2G081930, NACTF36) and ANAC087
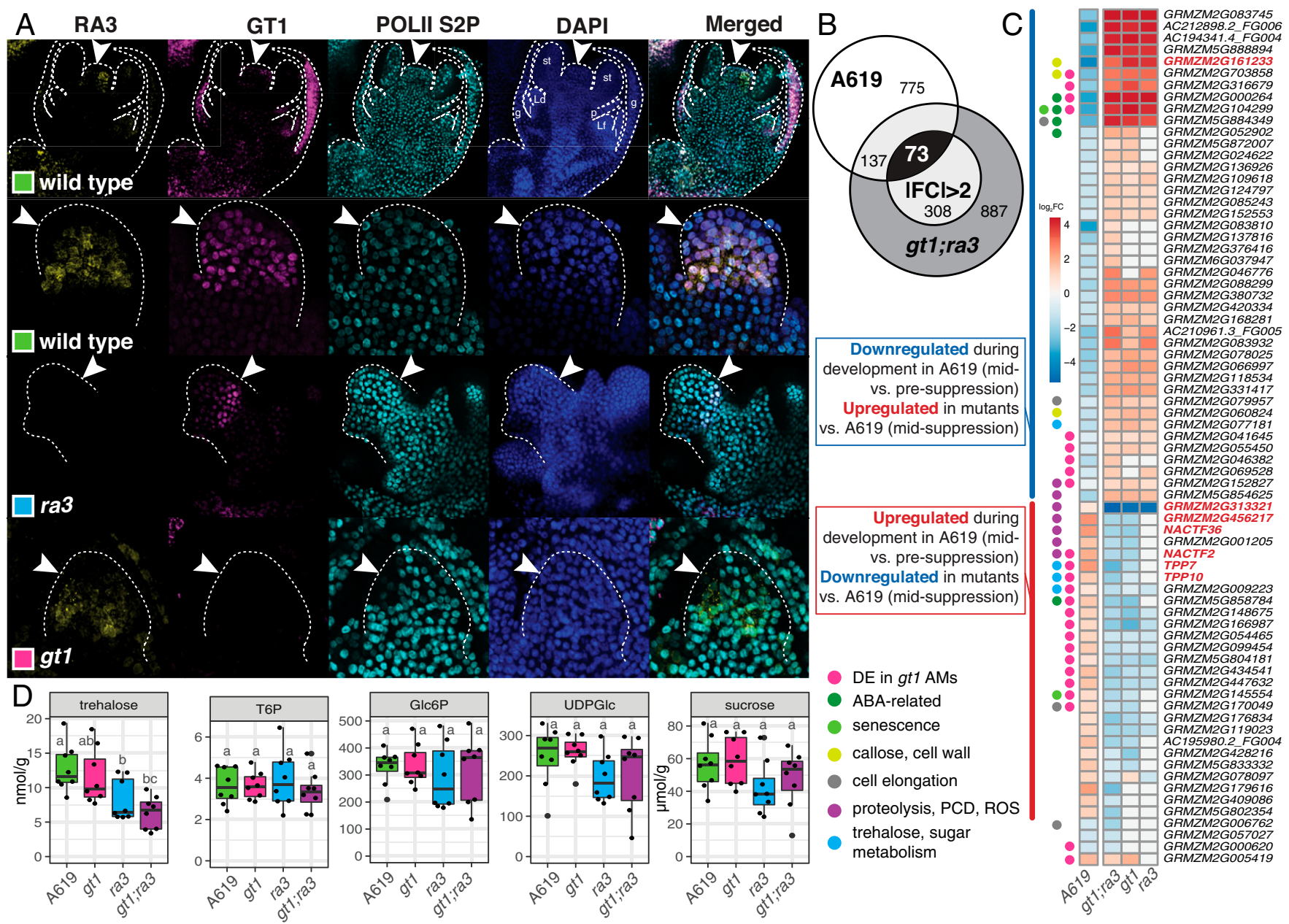
development in A619 (midvs. pre-suppression Downregulated in mutants vs. A619 (mid-suppression)

Fig. 4. GT1 and RA3 proteins localized to nuclei of carpels in tassel flowers, where trehalose metabolism is impacted in gt1; ra3 mutants. ( $A$ ) Fluorescent immunolocalizations of GT1, RA3, and aPOLII proteins using native antibodies in wild-type (B73), gt1, and ra3 mutants. Secondary antibodies were labeled with Alexa 488 (aRA3), Alexa 647 (aGT1), or Alexa 568 (aPOL II). g, glume; I, lemma; Ld, lodicule; Lf, lower flower; p, palea; st, stamen. Arrowheads indicate carpel primordia. (B) 73 genes changed over wild-type carpel development and were misexpressed in gt1;ra3 mutant flowers (|fold change [FC] $>2$ ). (C) The set of 73 carpel suppression candidate genes includes genes with roles in proteolysis, programmed cell death (PCD), and trehalose metabolism. 26 of the 73 carpel suppression candidate genes are also misexpressed in gt1 axillary meristems (AMs) (45). Genes discussed in text in red. ABA, abscisic acid; ROS, reactive oxygen species. (D) T6P and sucrose levels were not significantly different between A619 and mutant tassels. Trehalose was significantly lower in gt1;ra3 tassels. Different letters indicate statistically significant differences (eight tassels per genotype, $P<0.05$, ANOVA, Tukey's post hoc). 
(GRMZM2G181605, NACTF2) were up-regulated as carpel suppression commenced in A619 but expressed at lower levels in mutant tassel primordia versus A619 at the midsuppression stage (Fig. $4 D)(33,34)$. KIRA1 acts to regulate programmed cell death in stigmatic papillae (33). ANAC087, with ANAC046, initiates programmed cell death in the root cap and regulates chromatin degradation following programmed cell death (34). Two protease genes show a similar pattern of regulation: a cysteine protease gene (GRMZM2G456217) with an Arabidopsis homolog $(C E P 1)$ with roles in programmed cell death of xylem elements and the tapetum $(35,36)$ and an uncharacterized serine protease gene (GRMZM2G313321; see ref. 37). One of the genes that was down-regulated over development in A619 but highly up-regulated in mutant versus A619 tassels (GRMZM2 G161233) likely encodes a pectin biosynthetic enzyme involved in cell wall remodeling (38). Cell wall remodeling genes are also differentially expressed between upper and lower floral meristems in maize ears, and pectin modification differs between upper and lower floral ear meristems and between B73 and gt1;ra3 ear primordia (39). These and other examples (Table 1) are consistent with roles for $G T 1$ and $R A 3$ in carpel suppression and further point to genes that may be directly or indirectly regulated by GT1 and RA3.

Trehalose Levels Are Lower in gt1;ra3 Mutant Tassels. Two ra3 paralogs, TPP7 (GRMZM2G080354) and TPP10 (GRMZM2 $G 055150$ ), were highly up-regulated during carpel suppression in A619 and strongly down-regulated in gt1;ra3 mutants (Fig. $4 C$ and Table 1). Although RA3's enzymatic activity is not essential in regulating meristem determinacy, it is a catalytically active TPP enzyme (22). TPP enzymes catalyze the second (and last step) of the only trehalose biosynthesis pathway in plants; they dephosphorylate T6P to produce trehalose and a free phosphate group $(22,40)$. The misexpression of multiple TPP genes led us to measure sugar and metabolite levels at the carpel suppression stage in tassel primordia (1-1.5 cm in length) (Fig. 4D; SI Appendix, Fig. S4). Consistent with the downregulation of TPP genes, trehalose was substantially lower in gt1,ra3 mutants (Fig. 4D). However, T6P levels were not concomitantly higher in mutants versus A619. T6P is derived from the hexose phosphates glucose 6-phosphate (G6P) and uridine phosphate-glucose (UDPG) and likely signals sucrose status during plant growth and development (40-42). Similar to T6P and most metabolites, sucrose, G6P, and UDPG levels were also not significantly different between A619 and mutant tassels (Fig. 4D; SI Appendix, Fig. S4). This indicates that while trehalose biosynthesis is impacted in gt1; ra3 double mutants, sucrose status, as signaled by T6P $(40,42)$, is not affected, at least not at the level of entire tassel primordia. We reasoned that T6P levels may be unchanged because of the homeostatic control of sucrose and T6P levels (41). In support, four T6P synthase genes (TPSs), including at least one encoding a likely catalytically active TPS $(43,44)$, were also down-regulated in gt1;ra3 double mutants (Dataset S1). Similarly, trehalose metabolism gene expression and sugar accumulation differ between the upper and lower floral meristems in ear primordia (39). Taken together, these data suggest that the trehalose pathway is important in the regulation of carpel suppression.

ra3 Enhances Tillering in a gt1 Mutant Background. T6P signaling has roles in regulating bud outgrowth in eudicots $(45,46)$ and is associated with bud outgrowth in maize (47). Notably, 24 of

Table 1. Putative carpel suppression genes that have been functionally characterized*

\begin{tabular}{|c|c|c|c|c|}
\hline Maize gene $\mathrm{ID}^{\dagger}$ & Arabidopsis best hit & Functional annotation & Biological function & Citation \\
\hline GRMZM2G456217 & AT5G50260 & Cysteine protease & Programmed cell death & $(35,36)$ \\
\hline GRMZM2G081930 & AT4G28530 (KIRA1) & NAC transcription factor 36 (NACTF36) & Programmed cell death & (33) \\
\hline GRMZM2G181605 & AT5G18270 (ANAC087) & NAC transcription factor (NACTF2) & Programmed cell death & (34) \\
\hline GRMZM2G313321 & AT5G36210 & Serine protease, vignain & Proteolysis & $(37)$ \\
\hline GRMZM2G085243 & AT1G16470 (PAB1) & Proteasome subunit PAB1 & Proteolysis & \\
\hline GRMZM2G145554 & AT4G18170 (WRKY28) & WRKY DNA-binding domain protein & Leaf senescence & (98) \\
\hline GRMZM2G104299 & AT2G33620 & AT-hook motif nuclear-localized protein 10 & ABA response; senescence (maize) & $(99,100)$ \\
\hline GRMZM5G858784 & AT1G30100 (NCED5) & Nine-cis-epoxycarotenoid dioxygenase 3 & ABA biosynthesis & $(101)$ \\
\hline GRMZM2G052902 & AT2G24360 (RAF22) & Serine/threonine-protein kinase HT1 & ABA signaling & $(102)$ \\
\hline GRMZM5G884349 & AT2G35530 (bZIP16) & bZIP transcription factor protein (bZIP32) & $\mathrm{ABA}$ and $\mathrm{GA}$ response; cell elongation & $(103)$ \\
\hline GRMZM2G000264 & AT5G62670 (AHA11) & ATPase 4 plasma membrane-type & $A B A$ response & $(104)$ \\
\hline GRMZM2G088299 & AT3G01490 (CBC1) & MAP kinase kinase kinase 55 & Stomatal opening & $(105)$ \\
\hline GRMZM2G001205 & AT5G59820 (ZAT12) & ZNF5; $\mathrm{C} 2 \mathrm{H} 2$ zinc finger protein5 & ROS response; salt and osmotic stress & $(106-108)$ \\
\hline GRMZM2G152827 & AT5G14040 (PHT3;1) & Mitochondrial phosphate transporter 1 (MPT1) & ROS accumulation; salt stress & $(109,110)$ \\
\hline GRMZM5G854625 & AT1G60940 (SNRK2-10) & SNF1-related protein kinase 2.10 & ROS homeostasis; osmotic stress & $(111-115)$ \\
\hline GRMZM2G080354 & AT5G10100 (TPPI) & tpp10; trehalose-6-phosphate phosphatase 10 & Trehalose metabolism & $(116)$ \\
\hline GRMZM2G055150 & AT2G22190 (TPPE) & tpp7; trehalose-6-phosphate phosphatase 7 & Trehalose metabolism & $(117)$ \\
\hline GRMZM2G009223 & AT1G61800 (GPT2) & Glucose-6-phosphate/phosphate translocator & Response to sucrose & $(118)$ \\
\hline GRMZM2G077181 & AT3G57520 (SIP2) & Galactinol-sucrose galactosyltransferase 2 & Sugar metabolism & $(119)$ \\
\hline GRMZM2G376416 & AT1G02850 (BGLU11) & Beta-glucosidase 5 (GLU5) & Carbohydrat & \\
\hline GRMZM2G170049 & AT4G25560 (LAF1) & MYB domain protein 26 & Light signaling & $(120,121)$ \\
\hline GRMZM2G006762 & AT5G18280 (APY2) & Putative apyrase family protein & Cell elongation & $(122-124)$ \\
\hline GRMZM2G079957 & AT1G04240 (IAA3; SHY2) & Auxin-responsive protein IAA2 & & $(125-127)$ \\
\hline GRMZM2G161233 & AT3G23820 (GAE6) & UDP-glucuronate 4-epimerase 1 & Pectin biosynthesis; cell wall remodeling & (38) \\
\hline GRMZM2G060824 & AT5G23630 (MIA;PDR2) & ATPase PDR2 & Callose deposition; meristem maintenance & $(128)$ \\
\hline GRMZM2G703858 & AT3G25070 (RIN4) & RPM1-interacting protein 4 & Immunity; callose deposition & (129) \\
\hline GRMZM2G099454 & AT3G12500 (CHI-B) & Basic endochitinase & Immunity & $(130)$ \\
\hline GRMZM2G046382 & AT1G53540 & 17.4 kDa class I heat shock protein & Heat acclimation & $(131)$ \\
\hline GRMZM2G078025 & AT5G15450 (CLPB-P) & casein lytic proteinase B3 & Heat stress; plastid development & $(132,133$ \\
\hline GRMZM2G109618 & AT3G22880 (DMC1) & DMC1; disrupted meiotic cDNA homolog1 & Meiosis & $(134)$ \\
\hline
\end{tabular}

* Genes in the carpel suppression set of 73 genes with functional annotations, most of which are from Arabidopsis.

${ }^{\dagger}$ Gene IDs in bold differentially expressed in gt1 tiller buds (47). ABA, abscisic acid; cDNA, complementary DNA; GA, gibberellin; ROS, reactive oxygen species. 

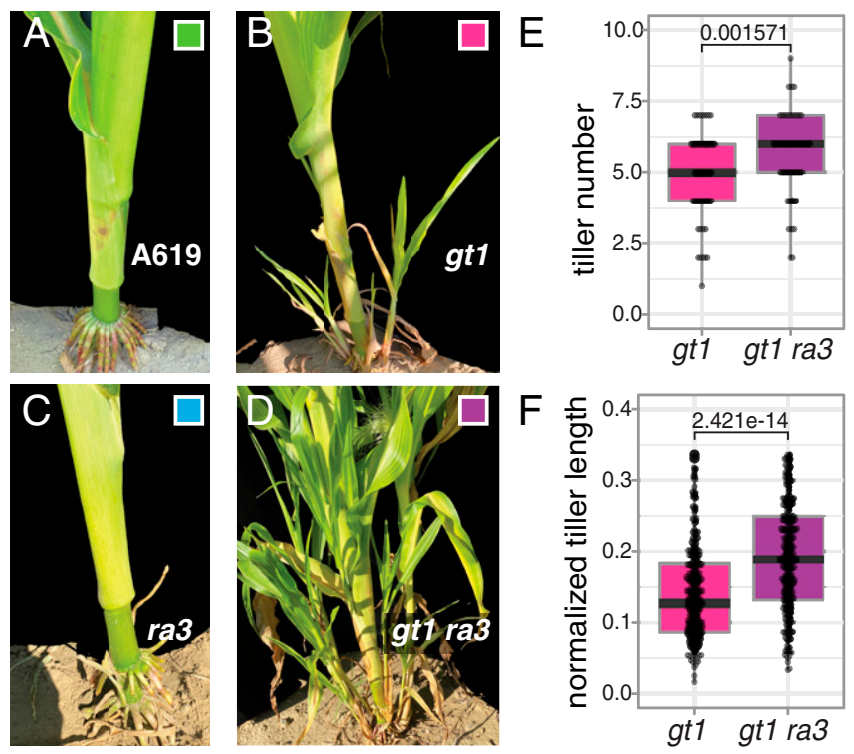

Fig. 5. GT1 and RA3 act in concert to regulate axillary meristem suppression. $(A-F)$ Tillering was enhanced in gt1;ra3 double mutants (57 gt1 and 64 gt1;ra3 individuals over two field seasons, $P<0.05$, ANOVA, Tukey's post hoc). gt1;ra3 double mutants produced both $(E)$ more and $(F)$ longer tillers. Tiller length in $F$ is normalized to the height of the main culm.

the 73 carpel suppression genes $(\sim 31 \%)$, including TPP7 and TPP10, were differentially expressed in $g t 1$ versus wild-type tiller buds (47). A gene set enrichment analysis also revealed that genes differentially expressed in $g t 1$ versus wild-type tiller buds (47) were enriched in the differentially expressed genes in our study (SI Appendix, Table S2), and RA3 and GT1 are bound and regulated by TEOSINTE BRANCHED1 (TB1), a conserved regulator of axillary bud suppression (47-49). These associations led us to ask whether $r a 3$ is also involved in regulating axillary bud outgrowth. We counted tillers and measured their lengths in A619, gt1, ra3, and gt1;ra3 plants and found that gt1;ra3 double mutants produced more and longer tillers than gt1 single mutants (Fig. 5). In addition, these double mutants produced more ears than single gt1 mutants (SI Appendix, Fig. S5). Thus, $R A 3$ also regulates vegetative branching in concert with GT1 and adds another developmental context in which both genes act to suppress growth.

\section{Discussion}

Organ repression is an important driving force in the evolution of floral diversity $(1,2)$. Here, we sought to identify the genes that regulate growth suppression in maize carpels and unexpectedly found the classic meristem determinacy gene, $R A 3$ (18). We show that $R A 3$ acts with $G T 1$ in multiple developmental contexts: to regulate carpel suppression, meristem determinacy, and vegetative branching. Although it is catalytically active, RA3's enzymatic activity is not essential for regulating meristem determinacy, and RA3 colocalizes with the transcriptional machinery in nuclear speckles in young ear primordia $(22,50)$. These data suggested that RA3 has an alternate "moonlighting" role in regulating transcription, potentially connected to T6P signaling $(22,50)$. Notably, we found a similar pattern of nuclear localization for both RA3 and GT1 in carpel primordia (Fig. 4A), suggesting RA3's moonlighting role extends to carpel suppression. Although the levels of T6P and its precursors were not significantly different between wild-type and mutant tassels, trehalose levels were lower in gt1; ra3 tassels, consistent with the down-regulation of other TPP genes (Fig. $4 C$ and $D$ ). These data suggest that the trehalose pathway, potentially connected to RA3's moonlighting role, has roles in regulating carpel suppression, as it does in a number of developmental processes (42).

The trehalose pathway and GT1-like genes have ancient roles in regulating vegetative branching. GT1-like transcription factors regulate axillary meristem growth suppression in Arabidopsis, maize, and Oryza sativa (rice) $(17,51,52)$. In addition, high T6P and sucrose levels lead to increased axillary bud outgrowth in Arabidopsis and Pisum sativum (garden pea) $(42,45)$. In maize, high T6P levels are correlated with axillary bud outgrowth in gt 1 and $t b 1$ mutants (47). Notably, we found that two independent mutations in $R A 3$ enhance $g t 1$ vegetative branching (Fig. 5; SI Appendix, Fig. S5). Taken together, these data indicate that growth regulation by the trehalose pathway and GT1-like genes appeared before the divergence of monocots and eudicots (Fig. 6).

In contrast to this ancient role in vegetative branching, carpel suppression arose later and repeatedly in the grasses, for example, in the lineages leading to maize and barley (Hordeum vulgare) $(3,53,54)$. Strikingly, floral organ suppression in barley is also mediated by a GT1 paralog, SIX-ROWED SPIKE1 (VRS1), and an $R A 3$ homolog is down-regulated in barley floral organ suppression mutants (54-59). Critically, while VRS1 does have a particular role in barley carpel suppression, it also acts to suppress the growth of other floral organs in lateral spikelets, and barley carpel suppression does not involve programmed cell death $(58,59)$. These differences in GT1 and VRS1 function, together with the evolutionary history of carpel suppression in the grasses, suggest that $R A 3$ and $G T 1$ homologs were independently recruited to mediate grass carpel suppression (Fig. 6). This recruitment may have been mediated by changes in expression of $G T 1$-like and trehalose metabolism genes (58, $60)$. Interestingly, in the eudicots, GT1-like genes have been independently recruited to mediate unisexual flower development in Diospyros kaki (persimmon; ref. 61), and a TPP gene occurs within the female-determining region of Vitis vinifera (grapevine; ref. 62). As in the grasses, floral unisexuality arose independently in the lineages leading to persimmon and grapevine, which are separated from each other by 120 million years and from the grasses by $\sim 160$ million years of evolution (63). This striking convergent evolution suggests that GT1-like genes and TPPs were repeatedly deployed to mediate growth repression in the development of unisexual flowers.

In conclusion, we show that $G T 1$-like and $R A 3$-like genes control an ancient growth suppression program recruited to multiple developmental contexts. This reflects the iterative

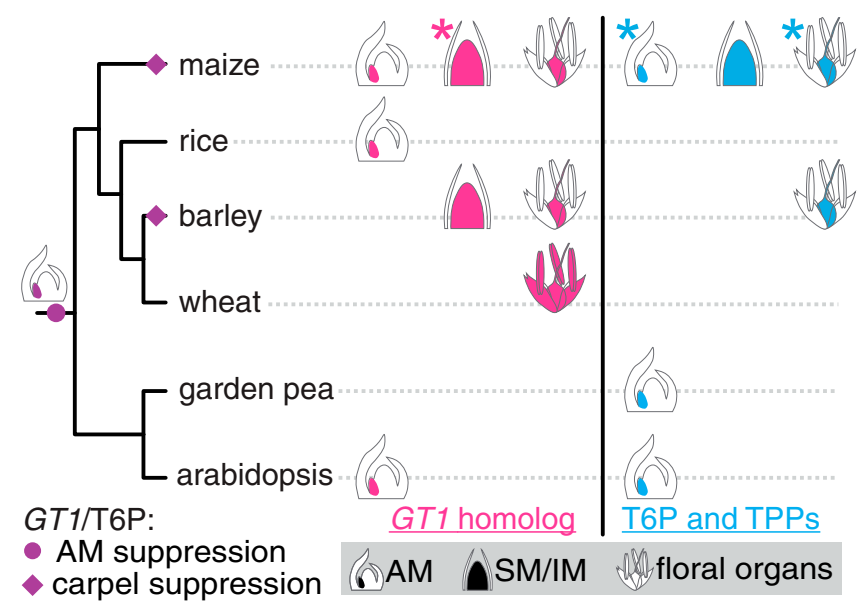

Fig. 6. GT1-like gene and trehalose pathway-mediated regulation of AM growth has been recruited to suppress carpel primordia. Asterisks are roles for GT1 and RA3 we show here; citations for roles of TPP genes and T6P are in the text. IM, inflorescence meristem; SM, spikelet meristem. 
nature of plant development, in which most aerial organs are leaf homologs, produced by a shoot apical meristem established early in embryogenesis (64-66). This deep homology of plant organs suggests that there may be additional cases of developmental genes seemingly functioning in discrete contexts, but whose pleiotropy is masked. Indeed, thorough genetic and phenotypic analyses continue to expose pleiotropy of developmental regulators (67-71). Recent examples include WUSCHEL-like HOMEOBOX9 (WOX9), which controls both inflorescence branching and embryo development (70), and THORN IDENTITY1, which controls thorn development and branching in citrus (71). Detailed dissection of gene functionalone, in the context of other genes, and in different developmental contexts-is likely to reveal that this pleiotropy is widespread.

\section{Materials and Methods}

Plant Material, Growth Conditions, and Phenotyping. We used the gt1-1 allele (17) for all our experiments with gt1. gt1-1 arose in A619 and was backcrossed five times with B73 to generate the B73 introgression lines used for BSA-seq and for phenotypic characterizations of the gt1;ra3 double mutants in B73. The ra3 alleles used here were either those that arose in our enhancer screen (ra3-rzl3, ra3-rzl4) in A619, or ra3-fea1 in B73 (18).

Plants for the ra3-rzl4 BSA-seq, gt1-1;ra3-rz/3 tassel and ear mature phenotypes, and gt1-1;ra3 tiller quantification were grown at the University of Massachusetts Amherst Crop and Animal Research and Education Farm in South Deerfield, MA $\left(\sim 42^{\circ} 29^{\prime} \mathrm{N}, 72^{\circ} 35^{\prime} \mathrm{W}\right)$. Plants for the gt1-1;ra3-fea 1 tassel and ear branch counts were grown over two seasons at the UMass Amherst Crop and Animal Research and Education Farm and near Valle de Banderas, Mexico $\left(\sim 20^{\circ} 47^{\prime} \mathrm{N}, 105^{\circ} 15^{\prime} \mathrm{W}\right)$. Plants for scanning electron microscopy (SEM), metabolite measurements, and RNA sequencing (RNA-seq) were grown in the College of Natural Sciences and Education Greenhouse on the UMass Amherst campus under long day conditions ( $16 \mathrm{~h}$ light, $8 \mathrm{~h}$ dark) at $28^{\circ} \mathrm{C}$.

Flowers and spikelets for phenotyping were selected from the central sections of ears or from the central sections of the tassel central spike. For tasse flower phenotyping, 20 flowers from each of four individuals per genotype were examined. For ear spikelet phenotyping, 50 spikelets from each of five individuals per genotype were examined. For measuring silk length, silks from 12 flowers from each of three individuals per genotype were measured. Tasse branches from 68 B73, $67 \mathrm{gt1}, 59 \mathrm{ra3}$, and $66 \mathrm{gt1}$; ra3 individuals and ear branches from 26 B73, $30 \mathrm{gt1}, 54 \mathrm{ra3}$, and $65 \mathrm{gt1}$; ra3 individuals were counted over two field seasons. Tillers were measured from $57 \mathrm{gt1}$ and $64 \mathrm{gt1}$; ra3 individuals in three planting blocks over the course of two field seasons. To account for differences in plant height, we normalized tiller length measurements to the height of the main culm. Statistical significance was assessed using an ANOVA and Tukey's test, $\chi^{2}$ test, or Student's $t$ test, as appropriate $(72,73)$

Enhancer Screen and gt1-1;rzl-4 BSA-seq. We performed an EMS mutagenesis screen as in (74). Briefly, gt1-1 (A619) pollen was mutagenized with EMS and crossed onto gt1-1 (B73) ears. M1 progeny were selfed to generate M2 families that were screened for enhanced silk growth in tassels. We identified $r z l-4$ and $r z l-3$ in this screen.

To map rzl-4, we crossed gt1-1;rzl-4 (A619) individuals to gt1-1 (B73) individuals and selfed the F1 progeny to generate an F2 mapping population. In the F2 population, leaves from 238 gt1-1;rzl-4 mutant individuals were selected for a pooled DNA extraction for BSA-seq, as described (19). Extracted genomic DNA was sequenced on an Illumina HiSEq. 2500 (paired-end reads, $150 \mathrm{bp})$ at Brigham Young University.

Bioinformatic tools on the Galaxy platform were used to assess read quality, align reads, and call SNPs and indels. We used FastQC (version 0.69) to assess read quality, and we used a PHRED cutoff of 20 (75). Reads were aligned to version 3 of the B73 reference genome using Bowtie2 (version 2.3.2.2) (76, 77). Variants were called using Samtools Mpileup (version 2.1.3) and filtered using Varscan (version 0.1) and Samtools filter pileup for SNPs and indels (78). Homozygous SNP variants were plotted using the R package ggplot2 (79) Candidate SNPs were identified using SnpEff (version 4.3a) (80), and SNPs of moderate effect were filtered using Provean (21).

Genotyping Assays. gt1-1 was genotyped with a cleaved amplified polymorphic sequences BsaJl restriction site that overlaps the gt1-1 lesion $(17,81)$ resulting in cleavage of the wild-type but not mutant allele. A 287-bp fragment surrounding the gt1-1 lesion was amplified with NEB Phusion High-Fidelity
DNA polymerase with GC buffer (New England Biolabs). PCR conditions were $98^{\circ} \mathrm{C}$ for $30 \mathrm{~s}, 72{ }^{\circ} \mathrm{C}$ for $15 \mathrm{~s}$, and $72{ }^{\circ} \mathrm{C}$ for $30 \mathrm{~s}$ ( 40 cycles). BsaJl cuts the $287-\mathrm{bp}$ fragment in the wild-type allele to 33 base pairs and 254 base pairs but does not cut the mutant gt1-1 allele.

ra3-rzl3 was genotyped with a derived cleaved amplified polymorphic sequences (dCAPS) Sau96I restriction site designed with dCAPS Finder 2.0 (82) (SI Appendix, Table S3). A 119-base pair fragment surrounding the ra3-rz/3 lesion was amplified with NEB Taq Polymerase with standard NEB buffer. PCR conditions were $95^{\circ} \mathrm{C}$ for $45 \mathrm{~s}, 60^{\circ} \mathrm{C}$ for $30 \mathrm{~s}$, and $68^{\circ} \mathrm{C}$ for $30 \mathrm{~s}$ ( 40 cycles). Sau96I cuts the 119-base pair fragment in the wild-type allele to 24 base pairs and 95 base pairs but does not cut the mutant ra3-rz/3 allele. PCR products were digested with BsaJl at $55^{\circ} \mathrm{C}$ for $1 \mathrm{~h}$ (gt1-1) or Sau96l at $37^{\circ} \mathrm{C}$ for $1 \mathrm{~h}$ (ra3$r z / 3)$ and run on a $3.5 \%$ agarose gels $(120 \mathrm{~V}$, for $60 \mathrm{~min})$ to separate the digested bands.

Protein Modeling. Protein homology modeling was performed with SWISSMODEL (83). A sequence of 363 amino acids encoded by the primary RA3 transcript was used as input into the homology search. RA3 had highest homology to the structure "Aspergillus fumigatus trehalose-6-phosphate phosphatase crystal form 1" (33.47\%, Protein Data Bank code: 5dxl, X-ray, $1.6 \AA$ ) (84). POLYVIEW-3d was used to visualize RA3 homology models with ra3-rz/4 (85). ConSurf was used to assess amino acid conservation (86).

Scanning Electron Microscopy. SEM was performed with a JEOL JCM-6000Plus Neoscope Benchtop scanning electron microscope with fresh tissue samples. Tassels and ears were dissected from the main culm and their bases painted with colloidal silver paint (Ted Pella, Inc.) and placed onto prechilled SEM stubs with electron microscope-conductive carbon double-sided tape (Nisshin). Stubs were prechilled on ice for at least $15 \mathrm{~min}$. Images were recorded under high vacuum and 5-kV voltage within 15 min of being in the SEM.

RNA-seq Library Preparation and Sequencing. We prepared a total of 32 pools of three tassels per pool at two developmental stages (1-1.1 cm and 1.3-2.0 $\mathrm{cm})$ for A619, gt1, ra3-rz/3, and gt1 ra3-rz/3 plants for RNA-seq. Pools for $g t 1$ and ra3-rzl3 samples contained a mix of individuals: either homozygous wildtype or heterozygous at ra3-rz/3 or gt1, respectively. Total RNA was extracted from 96 individual tassels using TRIzol and the Qiagen RNeasy Plant Mini kit protocol. Tissue was collected in $1.7 \mathrm{~mL}$ RNase-free safe-lock tubes with ceramic grinding beads and immediately placed into liquid nitrogen. Samples were ground in a Qiagen TissueLyser II for 30 seconds, and $1.0 \mathrm{~mL}$ of TRIzol was added to each sample and mixed well by vortexing until all sample powder was thoroughly mixed. Samples were incubated for five minutes at room temperature (RT), with frequent vortexing. Then, $0.2 \mathrm{~mL}$ of chloroform was added to each tube and vortexed for $15 \mathrm{~s}$, followed by a 1-min incubation at RT and another 15-s vortex. Samples were centrifuged at $15,000 \times g$ for 10 min to separate phases. After centrifugation, $200 \mu \mathrm{L}$ of the aqueous layer was removed and added to $700 \mu \mathrm{L}$ of Qiagen RLT buffer in a new tube. RLT buffer was prepared by adding $10 \mu \mathrm{L}$ of $\beta$-mercaptoethanol per $1 \mathrm{~mL}$ RLT buffer. Then, $500 \mu \mathrm{L}$ of $96-100 \%$ ( $\mathrm{vol} / \mathrm{vol}$ ) ethanol was added to the $200 \mu \mathrm{L}$ of sample now combined with $700 \mu \mathrm{L}$ RLT buffer. Samples were mixed well by vortexing, and half of the sample $(\sim 700 \mu \mathrm{L})$ was applied to a Qiagen MinElute pink spin column. From this point, samples were processed using the Qiagen RNeasy Plant Mini kit protocol, according to the manufacturer's instructions. RNA quality was assessed on a $1 \%$ agarose gel run for $20 \mathrm{~min}$ at $120 \mathrm{~V}$ and quantified using a Nanodrop 2000 spectrophotometer (Thermo Fisher Scientific). Four pools were generated for each genotype and developmental stage by combining $1 \mu \mathrm{g}$ of RNA from each of the three tassels. Total RNA from each sample was treated on-column with DNase I (NEB) for $15 \mathrm{~min}$. RNA pools were sent to Novogene for library construction and $20 \mathrm{Mbp}$ Illumina paired-end 150-bp sequencing for each pool.

RNA-seq Data Analysis. RNA-seq sequencing libraries were trimmed using Trimmomatic (version 0.36.3) (87). These were then aligned to the $Z$. mays B73 v3 genome (76) and processed into BAM files using STAR (version 2.7.0) (88). Read counts were generated with the Rsubread package function featureCounts in $\mathrm{R}(89,90)$. edgeR was used to construct principal component analysis plots of libraries (32). Because two of these libraries did not cluster with their corresponding replicates, we removed these libraries from further analysis (SI Appendix, Table S4).

Pairwise differential expression was calculated between wild-type and each mutant using edgeR (32). GO term enrichment was called using topGO (91) and maize-GAMER gene annotation (92). Enrichment of differentially expressed genes between wild-type and gt1 from Dong et al. (47) in this study was assessed via gene set enrichment analysis, with 1,000 permutations of the gene set in all cases except for the gt1 versus A619 postcomparison, which used 10,000 permutations (93). 
Antibody Purification. GT1 (guinea pig) and RA3 (rabbit) antisera were used for antibody affinity purification to the carboxyl-terminal region of GT1 or to the $\mathrm{N}$-terminal region of RA3 recombinant protein, using magnetic beads (Invitrogen), as described in (94). Validation of antibody was carried out by immunoblot following the protocol in (95), using total protein extract from wild-type and gt 1 or ra3 mutants as negative controls.

Immunolocalizations. To perform triple whole-mount immunolocalizations, we used previously published protocols with minor modifications $(50,96)$. Tassels between 1 and $1.5 \mathrm{~cm}$ from ra3-fea1 (B73), gt1-1 (B73), and $\mathrm{B} 73$ were collected and prefixed at $4{ }^{\circ} \mathrm{C}$ in $4 \%$ (wt/vol) paraformaldehyde and $2 \%$ ( $\mathrm{vol} / \mathrm{vol}$ ) Tween-20 in phosphate-buffered saline (PBS) for $1 \mathrm{~h}$, embedded in $6 \%$ agarose, sectioned at $75 \mu \mathrm{m}$ using a vibratome (Leica), and collected in fixative solution for $2 \mathrm{~h}$. Sections were washed and permeabilized by cell wall digestion ( $1 \%$ Driselase, Sigma-Aldrich; $0.5 \%$ cellulase, Sigma-Aldrich; $0.75 \%$ Pectolyase Y-23, Duchefa Bioche$\mathrm{mie}$ ) for $12 \mathrm{~min}$ at RT. Tissue was rinsed and incubated for $2 \mathrm{~h}$ in PBS and $2 \%$ Tween-20, rinsed two times, and blocked with $4 \%$ (wt/vol) bovine serum albumin (Sigma-Aldrich) for $1 \mathrm{~h}$. The blocking solution was removed, and the tissue sections were incubated overnight at $4{ }^{\circ} \mathrm{C}$ with anti-RA3 (1:200), anti-GT1 (1:75), and anti-YSPTSPS repeat S2Pho (RNA Pol II, B1 subunit; 1:200; Diagenode). The samples were washed for $8 \mathrm{~h}$ at $4{ }^{\circ} \mathrm{C}$ with gentle agitation with PBS $(0.2 \%$ vol/vol Tween-20), replacing the buffer every $2 \mathrm{~h}$, and incubated overnight at $4{ }^{\circ} \mathrm{C}$ with the appropriate secondary antibodies (Thermo Fisher Scientific) - anti-rabbit Alexa 488 (RA3), anti-mouse Alexa 568 (RNA POL II), and anti-guinea pig Alexa 647 (GT1)-counterstained with DAPI (Sigma), and mounted with ProLong Gold (Thermo Fisher Scientific). Immunolocalizations were repeated

1. L. Ronse De Craene, Understanding the role of floral development in the evolution of angiosperm flowers: Clarifications from a historical and physico-dynamic perspective. J. Plant Res. 131, 367-393 (2018)

2. P. J. Rudall, R. M. Bateman, Evolution of zygomorphy in monocot flowers: Iterative patterns and developmental constraints. New Phytol. 162, 25-44 (2004).

3. E. A. Kellogg, Flowering Plants. Monocots: Poaceae (Springer International Publishing, 2015).

4. S. Sakuma, T. Schnurbusch, Of floral fortune: Tinkering with the grain yield potential of cereal crops. New Phytol. 225, 1873-1882 (2020).

5. J. Friedman, S. C. H. Barrett, Wind of change: New insights on the ecology and evolution of pollination and mating in wind-pollinated plants. Ann. Bot. 103, 1515-1527 (2009).

6. L. Chen, Y.-G. Liu, Male sterility and fertility restoration in crops. Annu. Rev. Plant Biol. 65, 579-606 (2014).

7. P. C. Cheng, R. I. Greyson, D. B. Walden, Organ initiation and the development of unisexual flowers in the tassel and ear of Zea mays. Am. J. Bot. 70, 450-462 (1983).

8. A. Calderon-Urrea, S. L. Dellaporta, Cell death and cell protection genes determine the fate of pistils in maize. Development 126, 435-441 (1999).

9. A. DeLong, A. Calderon-Urrea, S. L. Dellaporta, Sex determination gene TASSELSEED2 of maize encodes a short-chain alcohol dehydrogenase required for stagespecific floral organ abortion. Cell 74, 757-768 (1993).

10. I. F. Acosta et al., tasselseed1 is a lipoxygenase affecting jasmonic acid signaling in sex determination of maize. Science 323, 262-265 (2009).

11. N. B. Best et al., nana plant2 encodes a maize ortholog of the Arabidopsis brassinosteroid biosynthesis gene DWARF1, identifying developmental interactions between brassinosteroids and gibberellins. Plant Physiol. 171, 2633-2647 (2016).

12. G. Chuck, R. Meeley, E. Irish, H. Sakai, S. Hake, The maize tasselseed4 microRNA controls sex determination and meristem cell fate by targeting Tasselseed6/indeterminate spikelet1. Nat. Genet. 39, 1517-1521 (2007).

13. C. Lunde, A. Kimberlin, S. Leiboff, A. J. Koo, S. Hake, Tasse/seed5 overexpresses a wound-inducible enzyme, ZmCYP94B1, that affects jasmonate catabolism, sex determination, and plant architecture in maize. Commun. Biol. 2, 114 (2019).

14. G. Chuck, R. Meeley, S. Hake, Floral meristem initiation and meristem cell fate are regulated by the maize AP2 genes ids1 and sid1. Development 135, 3013-3019 (2008).

15. S. E. Parkinson, S. M. Gross, J. B. Hollick, Maize sex determination and abaxial leaf fates are canalized by a factor that maintains repressed epigenetic states. Dev. Biol. 308, 462-473 (2007).

16. N. H. Nickerson, E. E. Dale, Tassel modifications in Zea mays. Ann. Mo. Bot. Gard. 42 , 195-211 (1955)

17. C. J. Whipple et al., grassy tillers1 promotes apical dominance in maize and responds to shade signals in the grasses. Proc. Natl. Acad. Sci. U.S.A. 108, E506-E512 (2011)

18. N. Satoh-Nagasawa, N. Nagasawa, S. Malcomber, H. Sakai, D. Jackson, A trehalose metabolic enzyme controls inflorescence architecture in maize. Nature 441, 227-230 (2006)

19. H. Klein et al., Bulked-segregant analysis coupled to whole genome sequencing (BSA-Seq) for rapid gene cloning in maize. G3 (Bethesda) 8, 3583-3592 (2018).

20. Z. Liang, J. C. Schnable, RNA-Seq based analysis of population structure within the maize inbred B73. PLoS One 11, e0157942 (2016). at least three times for each genotype. Images were acquired using a Zeiss LSM 780 confocal microscope.

Metabolite Measurements. Tassels between 1-1.4 cm were dissected from A619, gt1-1, ra3-rzl3;gt1-1/+, and gt1-1;ra3-rz/3 individuals and immediately frozen in liquid nitrogen. Eight tassels of each genotype were sampled in four waves over a 4-wk period. All individuals in a wave were sampled at $15 \mathrm{~h} 00 \mathrm{~min}$ to minimize variability. T6P and sugar metabolites were extracted from sin gle tassels using a protocol from (41). T6P, other phosphorylated intermediates, and organic acids were measured by anion-exchange high performance liquid chromatography coupled to tandem mass spectrometry as described by (41) with modifications as described by (97). Sugars and sugar alcohols were measured as described by (98). Statistical significance was assessed using an ANOVA and Tukey's test.

Data Availability. Raw sequencing data are available at the National Center for Biotechnology Information BioProjects (RNA-seq: PRJNA657042; ra3-rz/4 BSA-seq: PRJNA656888); all data underlying the figures are available either in SI Appendix or in Datasets S1 and S2.

ACKNOWLEDGMENTS. We thank members of the M.B. laboratory for thoughtful discussion and comments on this work and critical friends Zachary Lippman and Beth Thompson. We thank Amber De Neve, Ed Wilcox, Jae Hyung Lee, and Xiaosa Xu for help with experimentation and Dan Jones, Chris Joyner, Chris Phillips, and Neal Woodard for essential help with nursery management and plant care. This work was supported by the NSF (IOS-1652380 to M.B., IOS-1755141 to D.J.), the USDA National Institute of Food and Agriculture (NIFA-2019-67012-29654 to J.G., NIFA-2020-67013-31614 to G.C.), the University of Massachusetts, and the Max Planck Society.

21. Y. Choi, A. P. Chan, PROVEAN web server: A tool to predict the functional effect of amino acid substitutions and indels. Bioinformatics 31, 2745-2747 (2015).

22. H. Claeys et al., Control of meristem determinacy by trehalose 6-phosphate phosphatases is uncoupled from enzymatic activity. Nat. Plants 5, 352-357 (2019).

23. E. E. Irish, T. Nelson, Sex determination in monoecious and dioecious plants. Plant Cell 1, 737-744 (1989).

24. J. C. Kim et al., Cell cycle arrest of stamen initials in maize sex determination. Genet ics 177, 2547-2551 (2007).

25. L. Colombo, G. Marziani, S. Masiero, P. E. Wittich, BRANCHED SILKLESS mediates the transition from spikelet to floral meristem during Zea mays ear development. Plant J. 16, 355-363 (1998).

26. J. Strable, E. Vollbrecht, Maize $Y A B B Y$ genes drooping leaf1 and drooping leaf2 regulate floret development and floral meristem determinacy. Development 146 dev171181 (2019)

27. B. E. Thompson et al., bearded-ear encodes a MADS box transcription factor critical for maize floral development. Plant Cell 21, 2578-2590 (2009).

28. M. Mena et al., Diversification of C-function activity in maize flower development Science 274, 1537-1540 (1996).

29. E. Irish, Class II tassel seed mutations provide evidence for multiple types of inflorescence meristems in maize (Poaceae). Am. J. Bot. 84, 1502 (1997).

30. R. Reinheimer, F. O. Zuloaga, A. C. Vegetti, R. Pozner, Diversification of inflores cence development in the PCK clade (Poaceae: Panicoideae: Paniceae). Am. J. Bot 96, 549-564 (2009).

31. C. J. Whipple, Grass inflorescence architecture and evolution: The origin of nove signaling centers. New Phytol. 216, 367-372 (2017)

32. M. D. Robinson, D. J. McCarthy, G. K. Smyth, edgeR: A Bioconductor package for differential expression analysis of digital gene expression data. Bioinformatics 26 139-140 (2010).

33. Z. Gao et al., KIRA1 and ORESARA 1 terminate flower receptivity by promoting cell death in the stigma of Arabidopsis. Nat. Plants 4, 365-375 (2018).

34. M. Huysmans et al., NAC transcription factors ANAC087 and ANAC046 control distinct aspects of programmed cell death in the Arabidopsis columella and lateral root cap. Plant Cell 30, 2197-2213 (2018).

35. J. Han et al., The papain-like cysteine protease CEP1 is involved in programmed cell death and secondary wall thickening during xylem development in Arabidopsis. $J$. Exp. Bot. 70, 205-215 (2019).

36. D. Zhang et al., The cysteine protease CEP1, a key executor involved in tapetal programmed cell death, regulates pollen development in Arabidopsis. Plant Cell 26 2939-2961 (2014).

37. A. Tsuji, Y. Fujisawa, T. Mino, K. Yuasa, Identification of a plant aminopeptidase with preference for aromatic amino acid residues as a novel member of the prolyl oligopeptidase family of serine proteases. J. Biochem. 150, 525-534 (2011).

38. G. Bethke et al., Pectin biosynthesis is critical for cell wall integrity and immunity in Arabidopsis thaliana. Plant Cel/ 28, 537-556 (2016).

39. H. Yang, K. Nukunya, Q. Ding, B. E. Thompson, Tissue-specific transcriptomics reveal functional differences in maize floral development. Plant Physiol., 10.1093/plphys/ kiab557 (2021).

40. M. J. Paul, L. F. Primavesi, D. Jhurreea, Y. Zhang, Trehalose metabolism and signaling. Annu. Rev. Plant Biol. 59, 417-441 (2008). 
41. J. E. Lunn et al., Sugar-induced increases in trehalose 6-phosphate are correlated with redox activation of ADPglucose pyrophosphorylase and higher rates of starch synthesis in Arabidopsis thaliana. Biochem. J. 397, 139-148 (2006).

42. F. Fichtner, J. E. Lunn, The role of trehalose 6-phosphate (Tre6P) in plant metabolism and development. Annu. Rev. Plant Biol. 72, 737-760 (2021).

43. M.-L. Zhou et al., Trehalose metabolism-related genes in maize. J. Plant Growth Regul. 33, 256-271 (2014).

44. C. Henry et al., The trehalose pathway in maize: Conservation and gene regulation in response to the diurnal cycle and extended darkness. J. Exp. Bot. 65, 5959-5973 (2014).

45. F. Fichtner et al., Trehalose 6-phosphate is involved in triggering axillary bud outgrowth in garden pea (Pisum sativum L.). Plant J. 92, 611-623 (2017).

46. C. Tarancón, E. González-Grandío, J. C. Oliveros, M. Nicolas, P. Cubas, A conserved carbon starvation response underlies bud dormancy in woody and herbaceous species. Front Plant Sci 8, 788 (2017).

47. Z. Dong et al., The regulatory landscape of a core maize domestication module controlling bud dormancy and growth repression. Nat. Commun. 10, 3810 (2019).

48. M. Wang et al., BRANCHED1: A key hub of shoot branching. Front Plant Sci 10, 76 (2019).

49. J. Doebley, A. Stec, L. Hubbard, The evolution of apical dominance in maize. Nature 386, 485-488 (1997).

50. E. Demesa-Arevalo, M. J. Abraham-Juarez, X. Xu, Maize RAMOSA3 accumulates in nuclear condensates enriched in RNA POLYMERASE II isoforms during the establishment of axillary meristem determinacy. bioRxiv [Preprint] (2021). https://doi.org/10. 1101/2021.04.06.438639 (Accessed 30 October 2021)

51. E. González-Grandío et al., Abscisic acid signaling is controlled by a BRANCHED1 HD-ZIP / cascade in Arabidopsis axillary buds. Proc. Natl. Acad. Sci. U.S.A. 114, E245-E254 (2017).

52. V. Kumar et al., Tiller outgrowth in rice (Oryza sativa L.) is controlled by OsGT1, which acts downstream of FC1 in a PhyB-independent manner. J. Plant Biol. 64 417-430 (2021).

53. L. G. Le Roux, E. A. Kellogg, Floral development and the formation of unisexual spikelets in the Andropogoneae (Poaceae). Am. J. Bot. 86, 354-366 (1999).

54. R. Koppolu et al., Six-rowed spike4 (Vrs4) controls spikelet determinacy and rowtype in barley. Proc. Natl. Acad. Sci. U.S.A. 110, 13198-13203 (2013).

55. Y. Shang et al., A CYC/TB1-type TCP transcription factor controls spikelet meristem identity in barley. J. Exp. Bot. 71, 7118-7131 (2020).

56. N. Poursarebani et al., COMPOSITUM 1 contributes to the architectural simplification of barley inflorescence via meristem identity signals. Nat. Commun. 11, 5138 (2020).

57. J. Thiel et al., Transcriptional landscapes of floral meristems in barley. Sci. Adv. 7, eabf0832 (2021)

58. S. Sakuma et al., Divergence of expression pattern contributed to neofunctionalization of duplicated HD-Zip I transcription factor in barley. New Phytol. 197, 939-948 (2013).

59. M. Zwirek, R. Waugh, S. M. McKim, Interaction between row-type genes in barley controls meristem determinacy and reveals novel routes to improved grain. New Phytol. 221, 1950-1965 (2019).

60. D. M. Wills et al., From many, one: Genetic control of prolificacy during maize domestication. PLoS Genet. 9, e1003604 (2013).

61. T. Akagi, I. M. Henry, R. Tao, L. Comai, Plant genetics. A Y-chromosome-encoded small RNA acts as a sex determinant in persimmons. Science 346, 646-650 (2014).

62. C. Zou et al., Multiple independent recombinations led to hermaphroditism in grapevine. Proc. Natl. Acad. Sci. U.S.A. 118, e2023548118 (2021).

63. S. Kumar, G. Stecher, M. Suleski, S. B. Hedges, TimeTree: A resource for timelines, timetrees, and divergence times. Mol. Biol. Evol. 34, 1812-1819 (2017).

64. D. R. Kaplan, T. J. Cooke, Fundamental concepts in the embryogenesis of dicotyledons: A morphological interpretation of embryo mutants. Plant Cell 9, 1903-1919 (1997).

65. S. Leiboff, S. Hake, Reconstructing the transcriptional ontogeny of maize and sorghum supports an inverse hourglass model of inflorescence development. Curr. Biol. 29, 3410-3419.e3 (2019)

66. J. W. Von Goethe, G. L. Miller, The Metamorphosis of Plants (MIT Press, Cambridge, 2009).

67. S. A. Kessler et al., Conserved molecular components for pollen tube reception and fungal invasion. Science 330, 968-971 (2010).

68. C. Li et al., Glycosylphosphatidylinositol-anchored proteins as chaperones and co-receptors for FERONIA receptor kinase signaling in Arabidopsis. eLife 4, e06587 (2015).

69. T. Rosas-Diaz et al., A virus-targeted plant receptor-like kinase promotes cell-to-cel spread of RNAi. Proc. Natl. Acad. Sci. U.S.A. 115, 1388-1393 (2018).

70. A. Hendelman et al., Conserved pleiotropy of an ancient plant homeobox gene uncovered by cis-regulatory dissection. Cell 184, 1724-1739.e16 (2021).

71. F. Zhang et al., Reprogramming of stem cell activity to convert thorns into branches. Curr. Biol. 30, 2951-2961.e5 (2020)

72. S. M. Brady et al., Reassess the t test: Interact with all your data via ANOVA. Plant Cell 27, 2088-2094 (2015).

73. M. L. McHugh, The chi-square test of independence. Biochem. Med. (Zagreb) 23, 143-149 (2013).

74. M. G. Neuffer, "Mutagenesis" in The Maize Handbook, M. Freeling, V. Walbot, Eds (Springer, New York, 1994), pp. 212-219.
75. S. Andrews, FastQC: A quality control tool for high throughput sequence data. (2010). https://www.bioinformatics.babraham.ac.uk/projects/fastqd/ (Accessed 20 December 2021).

76. P. S. Schnable et al., The B73 maize genome: Complexity, diversity, and dynamics Science 326, 1112-1115 (2009)

77. B. Langmead, C. Trapnell, M. Pop, S. L. Salzberg, Ultrafast and memory-efficient alignment of short DNA sequences to the human genome. Genome Biol. 10, R25 (2009).

78. H. Li et al.; 1000 Genome Project Data Processing Subgroup, The sequence alignment/map format and SAMtools. Bioinformatics 25, 2078-2079 (2009).

79. H. Wickham, ggplot2: Elegant Graphics for Data Analysis. (Springer, 2016)

80. P. Cingolani et al., A program for annotating and predicting the effects of single nucleotide polymorphisms, SnpEff: SNPs in the genome of Drosophila melanogaster strain w1118; iso-2; iso-3. Fly (Austin) 6, 80-92 (2012).

81. G. P. Rédei, Ed., "CAPS (cleaved amplified polymorphic sequences)" in Encyclopedia of Genetics, Genomics, Proteomics and Informatics (Springer, Netherlands, 2008), pp. 270-270.

82. M. M. Neff, E. Turk, M. Kalishman, Web-based primer design for single nucleotide polymorphism analysis. Trends Genet. 18, 613-615 (2002).

83. A. Waterhouse et al., SWISS-MODEL: Homology modelling of protein structures and complexes. Nucleic Acids Res. 46 (W1), W296-W303 (2018).

84. Y. Miao et al., Structures of trehalose-6-phosphate phosphatase from pathogenic fungi reveal the mechanisms of substrate recognition and catalysis. Proc. Natl. Acad. Sci. U.S.A. 113, 7148-7153 (2016).

85. A. Porollo, J. Meller, Versatile annotation and publication quality visualization of protein complexes using POLYVIEW-3D. BMC Bioinformatics 8, 316 (2007)

86. H. Ashkenazy et al., ConSurf 2016: An improved methodology to estimate and visualize evolutionary conservation in macromolecules. Nucleic Acids Res. 44 (W1), W344-W350 (2016)

87. A. M. Bolger, M. Lohse, B. Usadel, Trimmomatic: A flexible trimmer for Illumin sequence data. Bioinformatics 30, 2114-2120 (2014)

88. A. Dobin et al., STAR: Ultrafast universal RNA-seq aligner. Bioinformatics 29, 15-21 (2013).

89. Y. Liao, G. K. Smyth, W. Shi, The R package Rsubread is easier, faster, cheaper and better for alignment and quantification of RNA sequencing reads. Nucleic Acids Res. 47, e47 (2019)

90. R. Ihaka, R. Gentleman, R: A language for data analysis and graphics. J. Comput. Graph. Stat. 5, 299-314 (1996).

91. A. Alexa, J. Rahnenfuhrer, topGO: Enrichment Analysis for Gene Ontology. R pack age version 2.44.0 (2016)

92. K. Wimalanathan, I. Friedberg, C. M. Andorf, C. J. Lawrence-Dill, Maize GO annotation-Methods, evaluation, and review (maize-GAMER). Plant Direct 2, e00052 (2018).

93. A. Subramanian et al., Gene set enrichment analysis: A knowledge-based approach for interpreting genome-wide expression profiles. Proc. Natl. Acad. Sci. U.S.A. 102 15545-15550 (2005).

94. G. S. Chuck, P. J. Brown, R. Meeley, S. Hake, Maize SBP-box transcription factors unbranched 2 and unbranched 3 affect yield traits by regulating the rate of lateral primordia initiation. Proc. Natl. Acad. Sci. U.S.A. 111, 18775-18780 (2014).

95. M. J. Abraham-Juárez, Western blot in maize. Bio 101, e3257 (2019)

96. T. M. Tran et al., An optimized whole-mount immunofluorescence method for shoot apices. Curr Protoc 1, e101 (2021).

97. C. M. Figueroa et al., Trehalose 6-phosphate coordinates organic and amino acid metabolism with carbon availability. Plant J. 85, 410-423 (2016).

98. R. Feil, J. E. Lunn, Quantification of soluble sugars and sugar alcohols by LC-MS/MS Methods Mol. Biol. 1778, 87-100 (2018).

99. T. Tian et al., Arabidopsis FAR-RED ELONGATED HYPOCOTYL3 integrates age and light signals to negatively regulate leaf senescence. Plant Cell 32, 1574-1588 (2020)

100. M. M. Wong et al., Phosphoproteomics of Arabidopsis highly ABA-Induced 1 identifies AT-Hook-Like10 phosphorylation required for stress growth regulation. Proc Natl. Acad. Sci. U.S.A. 116, 2354-2363 (2019).

101. R. S. Sekhon et al., Integrated genome-scale analysis identifies novel genes and networks underlying senescence in maize. Plant Cel/ 31, 1968-1989 (2019).

102. B.-C. Tan et al., Molecular characterization of the Arabidopsis 9-cis epoxycarotenoid dioxygenase gene family. Plant J. 35, 44-56 (2003)

103. Y. Kamiyama et al., Arabidopsis group C Raf-like protein kinases negatively regulate abscisic acid signaling and are direct substrates of SnRK2. Proc. Natl. Acad. Sci. U.S.A. 118, e2100073118 (2021)

104. W.-P. Hsieh, H.-L. Hsieh, S.-H. Wu, Arabidopsis bZIP16 transcription factor integrates light and hormone signaling pathways to regulate early seedling development. Plant Cell 24, 3997-4011 (2012).

105. X. Zhang et al., Inhibition of blue light-dependent $\mathrm{H}+$ pumping by abscisic acid through hydrogen peroxide-induced dephosphorylation of the plasma membrane H+-ATPase in guard cell protoplasts. Plant Physiol. 136, 4150-4158 (2004).

106. A. Hiyama et al., Blue light and $\mathrm{CO}_{2}$ signals converge to regulate light-induced stomatal opening. Nat. Commun. 8, 1284 (2017)

107. S. Davletova, K. Schlauch, J. Coutu, R. Mittler, The zinc-finger protein Zat12 plays a central role in reactive oxygen and abiotic stress signaling in Arabidopsis. Plant Physiol. 139, 847-856 (2005).

108. I. De Clercq et al., Integrative inference of transcriptional networks in Arabidopsis yields novel ROS signalling regulators. Nat. Plants 7, 500-513 (2021). 
109. L. Rizhsky, S. Davletova, H. Liang, R. Mittler, The zinc finger protein Zat12 is required for cytosolic ascorbate peroxidase 1 expression during oxidative stress in Arabidopsis. J. Biol. Chem. 279, 11736-11743 (2004).

110. F. Jia et al., Overexpression of mitochondrial phosphate transporter 3 severely hampers plant development through regulating mitochondrial function in Arabidopsis. PLoS One 10, e0129717 (2015).

111. W. Zhu et al., The mitochondrial phosphate transporters modulate plant responses to salt stress via affecting ATP and gibberellin metabolism in Arabidopsis thaliana. PLoS One 7, e43530 (2012).

112. H. Fujii, P. E. Verslues, J.-K. Zhu, Arabidopsis decuple mutant reveals the importance of SnRK2 kinases in osmotic stress responses in vivo. Proc. Natl. Acad. Sci. U.S.A. 108, 1717-1722 (2011).

113. F. Soma et al., ABA-unresponsive SnRK2 protein kinases regulate mRNA decay under osmotic stress in plants. Nat. Plants 3, 16204 (2017).

114. N. Fàbregas, T. Yoshida, A. R. Fernie, Role of Raf-like kinases in SnRK2 activation and osmotic stress response in plants. Nat. Commun. 11, 6184 (2020).

115. D. Kawa et al., SnRK2 protein kinases and mRNA decapping machinery control root development and response to salt. Plant Physiol. 182, 361-377 (2020).

116. K. P. Szymańska, L. Polkowska-Kowalczyk, M. Lichocka, J. Maszkowska, G. Dobrowolska, SNF1-related protein kinases SnRK2.4 and SnRK2.10 modulate ROS homeostasis in plant response to salt stress. Int. J. Mol. Sci. 20, 143 (2019).

117. Q. Lin, S. Wang, Y. Dao, J. Wang, K. Wang, Arabidopsis thaliana trehalose-6-phosphate phosphatase gene TPPI enhances drought tolerance by regulating stomatal apertures. J. Exp. Bot. 71, 4285-4297 (2020).

118. W. Wang et al., Trehalose-6-phosphate phosphatase E modulates ABA-controlled root growth and stomatal movement in Arabidopsis. J. Integr. Plant Biol. 62, 1518-1534 (2020).

119. B. C. Dyson, R. E. Webster, G. N. Johnson, GPT2: A glucose 6-phosphate/phosphate translocator with a novel role in the regulation of sugar signalling during seedling development. Ann. Bot. 113, 643-652 (2014).

120. J. Ma et al., The sucrose-regulated Arabidopsis transcription factor bZIP11 reprograms metabolism and regulates trehalose metabolism. New Phytol. 191, 733-745 (2011).

121. I.-C. Jang, R. Henriques, N.-H. Chua, Three transcription factors, HFR1, LAF1 and HY5, regulate largely independent signaling pathways downstream of phytochrome A. Plant Cell Physiol. 54, 907-916 (2013).
122. S. W. Yang, I.-C. Jang, R. Henriques, N.-H. Chua, FAR-RED ELONGATED HYPOCOTYL1 and FHY1-LIKE associate with the Arabidopsis transcription factors LAF1 and HFR1 to transmit phytochrome A signals for inhibition of hypocotyl elongation. Plant Cell 21, 1341-1359 (2009).

123. J. Wu et al., Apyrases (nucleoside triphosphate-diphosphohydrolases) play a key role in growth control in Arabidopsis. Plant Physiol. 144, 961-975 (2007).

124. C. Wolf, M. Hennig, D. Romanovicz, I. Steinebrunner, Developmental defects and seedling lethality in apyrase AtAPY1 and AtAPY2 double knockout mutants. Plant Mol. Biol. 64, 657-672 (2007).

125. G. Clark et al., Apyrase (nucleoside triphosphate-diphosphohydrolase) and extracellular nucleotides regulate cotton fiber elongation in cultured ovules. Plant Physiol. 152, 1073-1083 (2010).

126. Y. Xi et al., IAA3-mediated repression of PIF proteins coordinates light and auxin signaling in Arabidopsis. PLoS Genet. 17, e1009384 (2021).

127. C. Weiste et al., The Arabidopsis bZIP11 transcription factor links low-energy signalling to auxin-mediated control of primary root growth. PLoS Genet. 13, e1006607 (2017).

128. Q. Tian, J. W. Reed, Control of auxin-regulated root development by the Arabidopsis thaliana SHY2/IAA3 gene. Development 126, 711-721 (1999).

129. J. Müller et al., Iron-dependent callose deposition adjusts root meristem mainte nance to phosphate availability. Dev. Cell 33, 216-230 (2015).

130. E.-H. Chung, F. El-Kasmi, Y. He, A. Loehr, J. L. Dangl, A plant phosphoswitch platform repeatedly targeted by type III effector proteins regulates the output of both tiers of plant immune receptors. Cell Host Microbe 16, 484-494 (2014).

131. L. K. Hawkins et al., Characterization of the maize chitinase genes and their effect on Aspergillus flavus and aflatoxin accumulation resistance. PLoS One 10, e0126185 (2015).

132. N. Yamaguchi et al., H3K27me3 demethylases alter HSP22 and HSP17.6C expression in response to recurring heat in Arabidopsis. Nat. Commun. 12, 3480 (2021).

133. I. L. Parcerisa, G. L. Rosano, E. A. Ceccarelli, Biochemical characterization of ClpB3, a chloroplastic disaggregase from Arabidopsis thaliana. Plant Mol. Biol. 104, 451-465 (2020).

134. F. Myouga, R. Motohashi, T. Kuromori, N. Nagata, K. Shinozaki, An Arabidopsis chloroplast-targeted Hsp101 homologue, APG6, has an essential role in chloroplast development as well as heat-stress response. Plant J. 48, 249-260 (2006).

135. H. Chen et al., RAD51 supports DMC 1 by inhibiting the SMC5/6 complex during meiosis. Plant Cell 33, 2869-2882 (2021). 